\title{
Il fascino indiscreto del rito
}

\section{Cristiana Facchini}

Storicamente, 3 (2007).

ISSN: 1825-411X. DOI: 10.12977/stor550

II "caso Toaff", se così lo possiamo definire, è comprensibile entro una cornice di relazioni elementari, che compongono una trama di liasons dangereuses. II libro e le vicende che lo hanno accompagnato portano alla luce quesiti centrali per lo stato attuale della ricerca: il metodo dello storico e il ruolo della storia nella nostra società, i protocolli di controllo della ricerca storica, il rapporto tra storia e mezzi di comunicazione, i rapporti tra ricerca storica e case editrici.

Prima di tutto sarebbe opportuno chiedersi perché Toaff abbia scelto di pubblicare questa sua ricerca in Italia. Perchè in italiano? Perchè non in ebraico, la lingua della cultura in cui insegna da molti anni? Perchè non in inglese, la lingua scientifica internazionale?

Sono quesiti tutt'altro che secondari e lasciamo al lettore il compito di cercare le risposte.

Si è parlato di censura e pericolo per la libertà di ricerca. Ma a dire il vero le problematiche suscitate da questa vicenda sono di tutt'altro segno. Usciamo dal dibattito "politico" e rientriamo nei confini della critica legittima, quella scientifica, per vedere come interepretare meglio la vicenda.

Prendiamo quindi in mano il libro e analizziamone i contenuti, i dispositivi narrativi, e le fonti sulle quali la ricerca è stata condotta.

Non si può omettere l'analisi del paratesto che in questo caso è di assoluta centralità. La prima di copertina, il titolo, il sottotitolo, l'immagine che li accompagna e la quarta di copertina: i primi segni che un lettore più o meno 
interessato osserva immediatamente, in una libreria, se vuole sapere qualche cosa del libro.

Quarta di copertina. Non ci è dato di sapere se la breve sintesi che introduce il libro sia stata scritta da Toaff o sia una nota redazionale della casa editrice. Si dice che

questo libro affronta coraggiosamente uno dei temi più controversi nella storia degli ebrei d'Europa [...]; Rileggendo senza pregiudizi la documentazione antica di quel processo e di vari altri alla luce della più vasta situazione europea e anche di una puntuale conoscenza dei testi ebraici, l'autore mette in luce i significati rituali e terapeutici che il sangue aveva nella cultura ebraica, giungendo alla conclusione che, in particolare per l'ebraismo ashkenazita, l'accusa del sangue non era sempre un'invenzione.

Che fosse un tema controverso nella storia degli ebrei d'Europa ci pare curioso, perchè onestamente il tema non è mai stato controverso. Semmai la questione dell'omicidio rituale è tema controverso nella storia del cristianesimo e della chiesa, visto che è all'interno delle sue istituzioni che esso prende forma.

Per gli ebrei d'Europa l'accusa del sangue è uno dei tanti capi d'accusa con cui l'antisemitismo medievale costellava i suoi discorsi contro un nemico, non esclusivamente rappresentato dagli ebrei, ma anche da altri gruppi religiosi cosiddetti eretici. Ne sono testimonianza le apologie e le difese, le discussioni che cominciano ad essere elaborate nel corso del XVII secolo. In seconda istanza, la quarta di copertina sostiene che la documentazione antica del processo di Trento è stata riletta in modo innovativo presumibilmente privo di pregiudizi - ma soprattutto che a quelle fonti Toaff ne aggiunge di nuove che gli storici non ebrei non possono conoscere perchè non sono capaci di leggerle, le fonti ebraiche, vero e proprio luogo esoterico della storiografia recente. II luogo a cui hanno accesso pochi eletti. Dovremmo quindi immaginare che Toaff ci sveli e porti alla luce nuove fonti 
da sempre inaccessibili a chi non conosce l'ebraico.

Altro elemento menzionato nella quarta di copertina è relativo al sangue ma non si dice che tipo di sangue. Di animali, di esseri umani? Elemento terapeutico e rituale fondamentale, soprattutto per la cultura ebraica ashkenazita: l'unica per la quale l'accusa, forse, non è stata solo un'invenzione.

Prima di copertina: un titolo e un sottotitolo esplicitamente polemici, per non dire "scandalosi". Ognuno di noi sa quanto sia importante la scelta dei titoli. Pasque di sangue ha molteplici allusioni, ma per un pubblico italiano il termine Pasqua e il termine sangue costruiscono una connessione simbolica principalmente con il sangue del Cristo.

II sottotitolo: Ebrei d'Europa e omicidi rituali. Perchè un enunciato così generale, quando l'oggetto della ricerca è dedicato al caso di Simonino di Trento? Perchè proclamare una generalizzazione ricavata da un singolo evento? Non sarebbe stato appropriato un punto interrogativo, almeno nel titolo, che avrebbe evitato di enunciare già nella prima di copertina i risultati della ricerca?

Al titolo gibsoniano si accompagna un'immagine un po' rozza: un signore in abiti medievali, con tanto di barba e coltellaccio, sta per sgozzare un bambino coricato su di un letto di rovi. Un fuoco a lato e sopra una immagine naïve: un angelo che appare all'interno di una nube.

L'immagine commenta o meglio descrive il noto passo di Genesi 22. II brano di Genesi, noto nella cultura cristiana come sacrificio di Isacco, in ebraico è noto con il lemma di Aqedat yishaq, che ha una ricca tradizione ermeneutica nella cultura ebraica. La miglior traduzione è "La legatura di Isacco".

Abramo sta per "sacrificare" suo figlio perchè così gli ha chiesto il Signore. Ma quello stesso Dio che aveva messo alla prova la sua fede gli manda un messo, che appare tra le nuvole. II volto di Abramo è girato, ascolta la voce del messo divino. 
Ma l'Angelo dell'Eterno lo chiamò dal cielo e disse: «Abrahamo, Abrahamo!». Egli rispose: «Eccomi». L'Angelo disse: «Non stendere la tua mano contro il ragazzo e non gli fare alcun male; ora infatti so che tu temi Dio, poiché non mi hai rifiutato tuo figlio, I'unico tuo figlio». Allora Abrahamo alzò gli occhi e guardò; ed ecco dietro di lui un montone, preso per le corna in un cespuglio. Così Abrahamo andò, prese il montone e l'offerse in olocausto invece di suo figlio. (Gen 22,11-13) [1].

L'immagine è efficace. Quanti italiani riconoscono i dati elementari di quella immagine? Cosa è scomparso nella immagine utilizzata nella prima di copertina?

Veniamo ora alla narrazione della storia.

\section{Ouverture}

La prefazione contiene alcune affermazioni rilevanti. Toaff ci dice che ha scelto di analizzare il caso trentino perchè è l'unico che abbia prodotto ampia documentazione. E inoltre ci ripete che gli studi sull'accusa del sangue sono sempre stati condotti «sulle persecuzioni e sui persecutori, sulla loro ideologia e sulle loro presumibili motivazioni, sul loro odio verso gli ebrei, sul loro cinismo politico o religioso, sul loro astio xenofobo e razzista, sul loro disprezzo per le minoranze» (8). Toaff è sempre stato preoccupato dell'appiattimento della storia ebraica sulla storia dell'antisemitismo, così frequente in Italia.

I classici studi sull'accusa del sangue - da quelli di G. Langmuir a quelli di R. Po-Chia Sia e Diego Quaglioni - avrebbero, sia pur in modo accurato e originale, elaborato una visione di parte [2], ritenendo quelle fonti inaffidabili e riducendo al silenzio la storia ebraica. Condivisibile prospettiva quella di far parlare la storia ebraica, come una delle tante storie dell'Europa cristiana. È da questa preoccupazione che lo studio di Toaff prende forma, volto a mostrare l'altra faccia della medaglia, la versione ebraica dei fatti. Chi sono cioè questi ebrei vittime dell'accusa del sangue?

Toaff ripete più volte che il suo studio è scientifico, imparziale volto a 
dimostrare se nelle confessioni degli ebrei medievali è possibile ricostruire un dato di realtà che non sia il mero frutto dell'immaginario dei persecutori.

Volendo quindi concludere che gli omicidi, celebrati nel rito della Pasqua, non fossero soltanto miti, cioè credenze religiose diffuse e strutturate in maniera più o meno coerente, ma piuttosto riti effettivi propri di gruppi organizzati e forme di culto realmente praticate saremo chiamati a una doverosa prudenza metodologica. II fenomeno, una volta provata inequivocabilmente la sua presenza, dovrà essere collocato nel contesto storico, religioso e sociale, oltre che nell'ambiente geografico, dove avrebbe trovato presumibilmente espressione, con le sue peculiari caratteristiche irripetibili altrove (12-13).

Questa affermazione espone l'oggetto della ricerca, gli omicidi commessi durante la Pasqua ebraica non sono miti ma anche riti, questo è ciò che Toaff cercherà di dimostrare. Per farlo è necessario analizzare la struttura del mito e le fonti che ci permettono di ricostruire il rito. Vedremo come Toaff affronta questa delicata questione storiografica.

Poco dopo

\begin{abstract}
[...] dobbiamo tener presente che nelle comunità ebraiche di lingua tedesca il fenomeno, quando attecchirà, sarà in genere limitato a gruppi presso i quali tradizioni popolari, che nel tempo avevano aggirato o sostituito le norme rituali della halakhah ebraica, e consuetudini radicate, impregnati di elementi magici e alchemici, si sposavano in un micidiale cocktail con un fondamentalismo religioso violento e aggressivo (13).
\end{abstract}

Pur avvertendo il lettore che poi l'accusa di omicidio rituale si attivava ogni volta che scompariva un bambino in primavera e che essa venne a diffondersi e a radicarsi nell'Europa cristiana colpendo comunità ebraiche in ogni zona geografica e in ogni periodo storico, è chiaro che la prefazione annuncia in un certo senso la tesi di fondo del libro ma anticipa anche le 
conclusioni.

Bisogna premettere che, come ogni libro, Pasque di sangue non esce dal nulla, ma si colloca in un filone di studi relativamente noto. In particolare, i lavori che hanno ispirato questa ricerca vanno ricercati in due testi fondamentali, pubblicati in ebraico e in inglese, quelli di Yisrael Yaacov Yuval e di Elliot Horowitz [3]. In entrambi i casi, gli autori volevano portare all'attenzione degli storici alcuni elementi culturali della storia dell'ebraismo medievale, che a loro dire sono stati emarginati o sottaciuti, per non dire denegati, nella precedente storiografia ebraica. È rilevante sottolineare che questi studi partivano dall'idea di mostrare, o meglio portare alla luce, i livelli di violenza presenti anche all'interno delle comunità ebraiche, cercando in qualche modo di superare quella classica concezione della storia ebraica come lachrymose history tanto deprecata dai maggiori studiosi dell'ebraismo. Si tratta cioè di «normalizzare la storia ebraica», per usare una concezione cara, prima che agli studiosi sionisti, ad alcuni dei padri fondatori più radicali della Wissenschaft des Judentums. Non vedere gli ebrei solo come vittime della storia, ma anche come soggetti della medesima, coi loro vizi e le loro virtù. Ma più di ogni altra cosa, Yuval e Horowitz hanno indagato le forme della "violenza ebraica", simbolica o reale che fosse, innestandosi su di un dibattito storiografico che ha prodotti studi particolarmente suggestivi su temi quali rituali e violenza o rituali di violenza.

Per semplificare, il libro di Toaff risente di un clima culturale che è percorso da innovazioni storiografiche, e da accese critiche: un clima caratterizzato da una vivace libertà di ricerca che, per lo meno su questi temi, non è però giunto in Italia. Basterà notare - per tutti coloro che hanno gridato alla censura - che sia Horowitz che Toaff hanno condotto le loro ricerche pur insegnando all'Università di Bar Ilan, nota per essere un'università religiosa. E sarà opportuno sottolineare che anche il libro di Horowitz ha suscitato un acceso dibattito nella comunità scientifica internazionale.

Questo per dire che il lettore 'normale' è privato di un contesto di riferimento più preciso, e anche gli storici di professione non sono messi nelle 
condizioni di comprendere fino in fondo la provenienza di questo lavoro, la sua struttura, i suoi riferimenti.

Habent sua fata libelli, ancor più quando circolano e sono tradotti in altre culture.

Quale alter ego per lo storico?

Ci tiene l'autore a sottolineare che il suo lavoro sarà condotto secondo i crismi della ricerca scientifica - dati probanti, vaglio delle fonti, verifica, dimostrazione, ampio uso dell'erudizione.

Ogni conclusione in qualunque direzione si muova, dovrà essere dimostrata dopo aver vagliato e verificato sine ira et studio gli elementi che la sostengono con le fonti a disposizione, che siano in grado di confermarne o negarne in maniera persuasiva e cogente (14).

Il paratesto scientifico è imponente, tanto che la narrazione degli eventi deve essere accompagnata da una attenta lettura delle note, collocate alla fine del libro.

È già evidente fin dalle prime pagine il desiderio dell'autore di farsi romanziere, il che rende la lettura di un libro di storia certamente gradevole. I primi cinque capitoli sono segnati dalla descrizione minuziosa, suggestiva, accattivante e talvolta fastidiosa di personaggi che poi appariranno sui banchi d'accusa a Trento. Racconti incalzanti sulle remote vicende della nascita dell'accusa del sangue appaiono inseriti nell'intreccio in modo rapsodico, con voli dell'immaginazione storica da un luogo all'altro, da un periodo all'altro.

La discesa nel nord Italia degli ebrei di cultura tedesca è descritta in modo caustico, sprezzante. Loschi faccendieri, uomini privi di scrupoli, mercanti pronti alle più discutibili operazioni, che cercano di consolidare la vita degli insediamenti ebraici nelle zone della Repubblica veneta e del nord Italia. Uomini che, sopravvissuti e segnati dalle tragedie delle persecuzioni 
medievali, sono intraprendenti, avvezzi alle cospirazioni e alle congiure.

Ciò che però dovrebbe colpire il lettore non sono solo i toni talvolta da feuilleton coi quali vengono descritti gli attori della vicenda, ma l'uso delle fonti che ritroviamo nelle note. Così come per altri temi rilevanti nell'economia della narrazione degli eventi, Toaff sceglie una strategia di indistinta selezione delle fonti e di conseguenza un incauto utilizzo dei dati che contengono. Non è un caso, e si può dimostrare, che tutte le informazioni curiose e anedottiche sui personaggi (come vestivano, il colore dei capelli, strane idiosincrasie), quelle che rendono suggestivo il racconto, le storie di cospirazioni o dettagli della quotidianità, provengano da fonti agiografiche, e cioè dalle informazioni raccolte nella prima metà del Settecento o anche nel primo Novecento per consolidare il culto del beato Simonino.

Sia il testo del Bonelli che quello del Divina sono utilizzati da Toaff senza alcuna precauzione, anzi tutto ciò che vi è contenuto è preso alla lettera, come se queste opere non fossero di per sè dense di concezioni culturali del periodo in cui furono scritte: la prima, redatta nel 1747, non può che risentire di una precisa corrente della storiografia e della agiografia cattoliche, le quali hanno destinato la raccolta erudita dei dati al consolidamento del culto dei bambini martirizzati. Di qualche anno successiva sarà la nota Relazione Ganganelli sull'accusa di omicidio rituale, che va menzionata come manifestazione di una diversa tendenza religiosa presente nel mondo cattolico del tempo [4].

Lo stesso vale per il testo di Divina, che Toaff utilizza nel medesimo modo: come aveva già osservato Diego Quaglioni nella introduzione alla raccolta dei documenti del processo tridentino, il testo risente della ampia diffusione dell'antisemitismo politico di matrice cattolica nei territori asburgici a cavallo tra Otto e Novecento.

Periodo in cui l'ideologia dell'antisemitismo cattolico viene arricchendosi di temi nuovi, di accenti e sfumature inedite, le quali accompagnano una recrudescenza della riattivazione del mito dell'omicidio rituale [5].

Toaff ritiene queste due agiografie utili perchè «scientifiche», e le usa senza 
alcuna precauzione. L'assunto di base è che se le fonti contengono molti dati allora sono affidabili perché "scientifiche". Secondo Toaff il dato che si trova nel documento dice la verità e la realtà. Cosa ovviamente falsa. Ma ciò che è ancor più interessante è notare come queste fonti diventino una sorta di alter ego dello storico.

Nel presentare i noti fatti tridentini l'avvincente respiro narrativo porta l'autore a prediligere dettagli di un certo tipo e ad ometterne altri, tra cui informazioni tutt'altro che secondarie. Un esempio di questa strategia selettiva la troviamo quando Toaff narra del ritrovamento dell'infante Simone, tralasciando di informare l'ingenuo lettore del fatto che fu proprio il rabbino della comunità ebraica a denunciare il ritrovamento del corpo. Toaff dimentica inoltre di dirci che in un primo momento l'uccisione del bambino fu attribuita ad un cristiano, Giovanni Schweizer, il quale venne sbrigativamente scagionato, mentre l'accusa si rivolgeva contro gli ebrei.

Certo, sono fatti noti (agli addetti ai lavori). Però, perché non presentarli al lettore? Lo storico dovrebbe non solo raccogliere indizi, fatti, prove, documenti, ma anche cercare di operare una serie di ipotesi e presentare diversi scenari. Ma tutto ciò romperebbe la coerenza di una narrazione volta a dimostrare ciò che già conosce.

Altrettanto interessante quanto discutibile è che Toaff scelga di non concedere la parola alle voci dissidenti, neppure a quella pur nota dell'inquisitore domenicano, Battista De' Giudici, mandato da papa Sisto IV a Trento per stendere un resoconto sulla regolarità della procedura processuale. Quando De' Giudici arriva a Trento - anche in questo caso i fatti sono noti - alcuni leader della comunità ebraica sono già stati giustiziati mentre altri membri, oltre alle donne e ai bambini, sono ancora incarcerati. Sono note anche le difficoltà incontrate da De' Giudici, che dovette riparare a Rovereto, in territorio veneziano, dove cercò di spostare la sede del processo; note sono pure le vicende che portarono alla stesura della sua Apologia. Questa fonte - forse perchè ritenuta "di parte" - non viene mai presentata. In qualche punto Toaff suggerisce che l'inquisitore domenicano 
non sia attendibile perchè al soldo degli ebrei che si erano attivati - come facevano in caso di pericolo - per difendere la comunità.

L'accusa rivolta all'inquisitore romano di essere corrotto dagli ebrei è ancora frutto dell'alter ego di Toaff, e cioè delle apologie cattoliche a cui attinge a piene mani.

\section{I riti degli ebrei}

Se la prima parte del libro mira ad interpretare in modo «innovativo» le deposizioni del processo di Trento - riportando al centro della interpretazione le voci degli apologeti - la parte più originale, quella annunciata come rivelatrice di nuove scoperte, è dedicata ai riti degli ebrei. Nei riti ebraici, territorio sconosciuto, fascinoso e perturbante nell'immaginario cristiano, si annidano le verità nascoste.

Vediamo allora come Toaff affronta la questione della ritualità ebraica, cosa vi scorga di così rilevante da poter rivelare una verità nascosta, la fondatezza dell'accusa del sangue rivolta agli ebrei ashkenaziti.

È a partire dal capitolo 6 che il registro analitico varia, per affrontare la cultura popolare ebraica. II capitolo che introduce questi temi - riti, cerimonie - è dedicato al ruolo che il sangue ricopriva nella cultura ebraica e crisiana di età medievale. Riflettendo in modo suggestivo le idee di Piero Camporesi (che però, bisogna ricordarlo, non era uno storico) Toaff compie una indagine sull'uso del sangue come emostatico e come potente strumento medico, in opposizione alle prescrizioni della tradizione normativa ebraica la halakhah. Se quindi vero è che la Bibbia proibisce il consumo e l'uso del sangue, Toaff dimostra che queste prescrizioni cadono sotto il peso della cultura. Così come i cristiani, gli ebrei non possono sottrarsi agli usi del tempo e usano il sangue essicato - animale e umano - per diversi fini. Non si tratta certo di informazioni o scoperte nuove: questi temi erano stati ampiamente descritti nei testi classici dedicati al folklore ebraico. E fino a questo punto, in linea di massima, potremmo essere d'accordo, se non fosse che anche in questo caso Toaff sembra essere guidato da un'ossessione. Non si limita a mostrare che anche gli ebrei usavano il 
sangue per curare malattie, per magia o altre cose, ma deve dimostrare qualche cosa in più, cioè che gli ebrei consumano e utilizzano sangue di bambino cristiano, in modo rituale.

Nessuna fonte dimostra questa sua tesi. Leggiamo cosa scrive.

Si potrebbe a questo punto concludere che il sangue di bambino in polvere, e soprattutto dei bambini cristiani, come emostatico nella circoncisione, visto il disinteresse nei suoi confronti anche da parte degli ebrei convertiti, per altri versi protesi a diffamare l'ebraismo, sia una chimera e una tendenziosa invenzione di inquisitori, ossessionati dal sangue, o degli stessi ebrei, terrorizzati dalle torture e disposti a compiacere pedissequamente i carnefici. Ma questa sarebbe una conclusione sbagliata e fuorviante (97).

Insomma, dopo aver cercato ovunque anche tra le fonti più discutibili, quelle dedicate ai riti ebraici redatte da ebrei convertiti (Morosini e il micidiale Paolo Medici) e non aver trovato alcunchè, nulla che gli permetta di dire che gli ebrei utilizzavano il sangue di bambino cristiano nei riti, Toaff abilmente invita il lettore a cercare altrove, a non farsi guidare da ciò che dicono le fonti che lui stesso presenta.

$\grave{E}$, questa, una strategia narrativa che si ritrova frequentemente nel testo. Allora, deve esistere qualche altro luogo della cultura ebraica che potrebbe fornire informazioni precise su questi riti segreti. E dove va a cercare queste fonti Toaff?

Ovviamente nella tradizione qabbalistica. È curioso però scoprire che a suggerire questo percorso sia proprio il principe vescovo Hinderbach, artefice del processo e della istituzionalizzazione del culto del beato Simonino. E sulla scia di Hinderbach e delle fonti apologetiche cattoliche che nella Qabbalah intravvedevano gli aspetti ancor più oscuri dell'ebraismo e dei suoi riti.

Non mi soffermo su questo aspetto, ma anche in questo caso Toaff non procura alcuna informazione nuova o sconosciuta sull'omicidio rituale. 
II capitolo 8 è dedicato alla festa di Purim e svolge una doppia funzione: mostrare gli usi della violenza nella festa "delle sorti" e mostrare la stretta connessione tra Purim e Pesach. In questo capitolo Toaff utilizza ampiamente i lavori do Elliot Horowitz.

Purim è una festività ebraica estremamente complessa per le diverse sfumature e per i diversi usi che essa può assumere e che di fatto ha assunto. Molto è stato scritto su questa festa, ma Toaff decide di presentare al suo lettore un solo aspetto del cosiddetto "carnevale ebraico".

L'assunto è quello di dimostrare che l'accusa di omicidio rituale "partiva da lontano". Nel caso di questo rito, dove l'arcinemico Aman viene sconfitto e distrutto, Toaff sostiene che l'uso di appendere o bruciare Aman (cosa del resto comune a tante feste popolari) ha significato simbolico traslato, cioè appendere e bruciare Gesù. Vediamo nel dettaglio la sua argomentazione.

Secondo il grande antropologo inglese James George Frazer, Cristo moriva mentre rappresentava Aman (il Dio morente) in un dramma di Purim nel quale (Gesù) Barabba, il doppio di Gesù di Nazareth, aveva recitato la parte di Mardocheo (il dio che risorge). Nel modello del dio che muore e rinasce, comune a tutto il vicino oriente, Aman rappresenterebbe la morte e Mardocheo la vita, mentre la celebrazione di Purim costituirebbe il rituale ebraico di morte e resurrezione. Muovendo da questa considerazione, si ipotizzerebbe che in passato gli ebrei, al culmine della festa, avessero il costume di mettere a morte un uomo in carne e ossa, e che Gesu fosse stato crocifisso in questo contesto, rappresentando il tragico ministro d Assuero e arcinemico di Israele (131-132).

E ancora, riprendendo Frazer, Toaff ripropone la tesi, che circolava nella prima età moderna, tratta dallo storico ecclesiastico Socrate Scolastico, nella quale si narra di un caso di brutale uccisione di un bambino cristiano avvenuto ad Inmestar in Siria nel 415 durante la festa di Purim (133). Secondo Toaff questo evento ha «tutti i crismi della veridicità», ma non ci spiega perchè. Il caso di Inmestar, fatto risalire al 415 dell'era cristiana, è poi 
associato a quello di Brie-Comte-Robert del 1191-92 (134), strutturalmente diverso, per mostrare che durante la festa di Purim gli ebrei potevano ammazzare un cristiano pecorrendo l'iter rituale della passione (135).

Poi, portando pochi esempi di vilipendio alla fede cristiana - offese alle immagini di Madonne, o forme varie di blasfemia - servono a Toaff per costruire una spiegazione "scientifica". Colpisce che Toaff non riesca a distinguere le forme diffuse di "offesa alla religione cristiana" che si trovano registrate negli incartamenti dei tribunali della prima età moderna, da riti veri e propri.

Inoltre l'ostinata ricerca di un nesso e una stretta relazione tra Purim e Pesach, risente di una lacuna metodologica abbastanza preoccupante per chi si dedichi all'analisi dei sistemi rituali.

Pare insomma che Toaff non abbia cognizione del dibattito sul rito che ha animato molta produzione scientifica in questi anni. Questa lacuna teorica si riflette nel modo in cui legge le fonti e in cui adotta il concetto di antirito, o misunderstanding rituale. II misunderstanding rituale che spiega, secondo Yuval e Toaff, la costruzione di riti ebraici modellati non tanto sulla tradizione normativa del giudaismo quanto sulle strutture rituali cristiane sarebbe una tesi interessante, e tutto sommato da prendere in considerazione, ma con l'avvertenza di avere una idea di che cosa sia un universo simbolico e rituale. Infine - sia nel caso di Toaff che in quello di Yuval e Horowitz - emerge un secondo dato problematico che ha a che fare con il metodo storico: abbagliati dalla affascinante quanto fantasiosa ipotesi di Frazer (che alla fine cercava di ricondurre il caso di Inmestar a precisi archetipi religiosi) tutti questi autori mostrano una certa leggerezza nell'affrontare le fonti antiche e tardo-antiche. Nel caso di Toaff poi, laddove si accetta la tesi della morte di Gesù come messa in scena di un rito di Purim, significa proprio cancellare l'imponente bibliografia e la raffinata metodologia analitica elaborata negli studi sul Gesù storico e sulla nascita del cristianesimo.

Lo stesso vale per il capitolo 12, dal significativo titolo /l memoriale della passione (173-188). II capitolo si apre ponendo al centro le voci degli 
imputati, visto che esse sono, secondo Toaff, affidabili, piene di informazioni dettagliate che hanno un immediato riscontro nella realtà.

Anche qui mancano le fonti probanti: indicativo è il modo in cui Toaff tratta la questione relativa al lemma ze ha-dam (questo è il sangue) che nella Haggadah di Pesach, il testo che si legge durante la cena pasquale, sarebbe collocato nella elencazione delle dieci piaghe. Ma gli artefici del processo avevano bisogno di una frase che desse un senso rituale diverso, avevano bisogno della frase ze ha-dam shel goi qatan, cioè «questo è il sangue del bambino cristiano». Una frase che Toaff non riesce a trovare in alcuna fonte e che viene spiegata - seguendo ancora una suggestione dei giudici e di Wolfang - attribuendola alla tradizione orale.

La narrazione in questo capitolo è caotica, contiene molti dati affastellati, non sempre facili da sintetizzare. Da un lato Toaff ritiene veritiera l'esistenza di un rito ebraico nel quale viene usato il sangue di bambino cristiano. Ma ci dice, sempre facendo parlare le fonti agiografiche, che non è un rito riconosciuto da tutti gli ebrei, neanche da tutti gli ebrei ashkenaziti. $\grave{E} a$ questo punto che immagina l'esistenza di un gruppo ristretto, i cosiddetti "fondamentalisti" ashkenaziti, che avrebbero trasmesso il rito in forma segreta, orale, per pochi eletti. Anche in questo caso viene il sospetto che i giudici volessero inserire, nell'articolata accusa di omicidio rituale, quella della cospirazione ebraica, che già era stata utilizzata nel periodo della peste [6].

Inoltre, detto en passant, Toaff utilizza fonti sui riti abbastanza peculiari. Da un lato mostra competenza nel trovare informazioni sugli usi rituali di diverse tradizioni ebraiche, diversi minhagim, ma talvolta privilegia testi discutibili. Molto spesso utilizza resoconti sui riti degli ebrei di età secentesca e settecentesca, così come le immagini che sceglie di antologizzare sono quasi tutte posteriori al periodo in questione.

Da un lato sarebbe stato opportuno riflettere sull'ampia pubblicazione di testi sui riti degli ebrei che occuparono gli eruditi d'Europa almeno a partire dal XVII secolo. Redatti in specifiche occasioni, generati da rapporti culturali 
complessi, i testi sui riti degli ebrei sono una fonte molto ricca, e stupisce che l'autore ne privilegi alcuni piuttosto che altri e non li tratti con la dovuta cautela.

Ma ciò che sconcerta è l'esegesi che ne fa. I testi non sono considerati come tali, ma come luogo in cui cercare la prova della propria ipotesi. I diversi livelli di significato del testo sono scomparsi, così come le glosse testuali e culturali ampiamente riconoscibili nelle confessioni rilasciate sotto tortura.

Ad un'attenta lettura questo libro mostra una costruzione semplice ma allo stesso tempo fortemente coesa. Utilizza le informazioni contenute negli atti processuali, nelle agiografie del Bonelli e del Divina, e sulla scia delle informazioni contenute in questi testi va alla ricerca di prove nelle fonti ebraiche per suffragare quelle informazioni. Le prove ebraiche sono trovate attraverso un esame superficiale e rapsodico di testi e fonti provenienti da zone geografiche e ambienti culturali diversi. L'interpretazione letterale di testi differenti per genere e composizione, appartenenti a periodo e luoghi diversi, redatti con funzionalità diverse è metodo di analisi alquanto problematico.

Alla fine la messe di dati raccolti è composta in una cornice di senso fortemente ipotetica, suggestiva, costruita per assonanze, somiglianze, veri e propri voli della immaginazione. Non certo una thick description, tanto meno una deep interpretation.

Il caso trentino è estremanente complesso per il tipo di fonti che ci ha lasciato, e per la sua ricca quanto controversa trama culturale e giuridica. Tutto questo poteva essere descritto e analizzato senza dover dimostrare a tutti i costi una particolare tesi. Anche adottando uno stile suggestivo, potevano essere salvaguardati i dubbi e le incoerenze, potevano essere presentate tutte le parti coinvolte, il mondo degli ebrei e quello dei cristiani. In ombra, se non addirittura cancellate, sono le caratteristiche della cultura cristiana del periodo, le battaglie tra diverse correnti teologiche, i conflitti tra 
gruppi religiosi a Trento e a Roma. E ancor più in ombra, la suggestiva quanto complessa vicenda che ha portato alla decostruzione del caso trentino, alla decostruzione cioè di un discorso ampiamente segnato dalla volontà di costruire un rito.

Ora tra le tante domande inevase, ci chiediamo: Ma cosa è successo a Giovanni Schweizer?

\section{Note}

[1] Bibbia di Diodati, a cura di M. Ranchetti, Milano, Mondadori, 1999.

[2] G. Langmuir, Toward a Definition of Antisemitism, Berkeley, University of California Press, 1990; R. Po-chia Hsia, Trent: Stories of a Ritual Murder, New Haven, Yale University Press, 1992; Ead., The Myth of Ritual Murder: Jews and Magic in Reformation Germany, New Haven, Yale University Press, 1988; Processi contro gli Ebrei di Trento, vol. 1., a cura di A. Esposito e D. Quaglioni, Padova, CEDAM, 1990.

[3] I. J. Yuval, Shene goyyim be-vitnekh: Yehudim we-notsrim dimuyim hadadiyim, Tel-Aviv, 'Am 'oved, 2000 poi tradotto in inglese, con parziale revisione dopo le critiche alla edizione ebraica, col titolo Two nations in your womb: perceptions of Jews and Christians in Late Antiquity and the Middle Ages, Berkeley, University of California Press, 2006; E. Horowitz, Reckless Rites: Purim and the legacy of Jewish Violence, Princeton, Princeton University Press, 2006.

[4] È curioso che Toaff non faccia mai riferimento alle problematiche relative alla nascita della cosiddetta storiografia barocca cattolica, in particolare sulla questione dei padri bollandisti. Alcuni cenni su queste problematiche si trovano in R. Taradel, L'accusa del sangue. Storia politica di un mito antisemita, Roma, Editori Riuniti, 2002; S. Zen, Baronio storico. Controriforma e crisi del metodo umanistico, Napoli, Vivarium, 1994; K. Stow, Jewish Dogs: an image and its interpreters, Stanford, Stanford 
University Press, 2006; T. Caliò, Antisemitismo e culto dei santi in età contemporanea: il caso del beato Lorenzino da Marostica, in: P. Golinelli (ed.), II pubblico dei santi: forme e livelli di ricezione dei messaggi agiografici , Roma, Viella, 2000.

[5] Si veda il romanzo di B. Malamud, L'uomo di Kiev, trad. it. Torino, Einaudi, 1997 (II ed.) ispirato al noto caso Beilis dei primi del Novecento. Ancora suggestiva l'interpretazione avanzata da F. Jesi, L'accusa del sangue , Brescia, Morcelliana, 1993, dedicato al caso di Damasco del 1840 su cui cfr. anche J. Frankel, The Damascus Affair. "Ritual Murder", Politics, and the Jews in 1840, Cambridge, Cambridge University Press, 1997.

[6] Si veda su questo argomento C. Ginzburg, Storia notturna: una decifrazione del sabba, Torino, Einaudi, 1995. 